\title{
The Genetic and Functional Basis of Purine Nucleotide Feedback-resistant Phosphoribosylpyrophosphate Synthetase Superactivity
}

\author{
Michael A. Becker, ${ }^{\star}$ Patrick R. Smith, ${ }^{\star}$ William Taylor, ${ }^{\star}$ Reba Mustafi, ${ }^{\star}$ and Robert L. Switzer ${ }^{\star}$ \\ ${ }^{*}$ Rheumatology Section, Department of Medicine, The University of Chicago, Chicago, Illinois 60637; and ${ }^{\ddagger}$ Department of Biochemistry, \\ University of Illinois, Urbana, Illinois 61801
}

\begin{abstract}
The genetic and functional basis of phosphoribosylpyrophosphate synthetase (PRS) superactivity associated with purine nucleotide inhibitor-resistance was studied in six families with this $\mathbf{X}$ chromosome-linked purine metabolic and neurodevelopmental disorder. Cloning and sequencing of PRS1 and PRS2 cDNAs, derived from fibroblast total RNA of affected male patients by reverse transcription and PCR amplification, demonstrated that each PRS1 cDNA contained a distinctive single base substitution predicting a corresponding amino acid substitution in the PRS1 isoform. Overall, the array of substitutions encompassed a substantial portion of the translated sequence of PRS1 cDNA. Plasmid-mediated expression of variant PRS1 cDNAs in Escherichia coli BL21 (DE3/pLysS) yielded recombinant mutant PRS1s, which, in each case, displayed a pattern and magnitude of purine nucleoside diphosphate inhibitor-resistance comparable to that found in cells of the respective patient. Kinetic analysis of recombinant mutant PRS1s showed that widely dispersed point mutations in the $X$ chromosomelinked PRPS1 gene encoding the PRS1 isoform result in alteration of the allosteric mechanisms regulating both enzyme inhibition by purine nucleotides and activation by inorganic phosphate. The functional consequences of these mutations provide a tenable basis for the enhanced production of phosphoribosylpyrophosphate, purine nucleotides, and uric acid that are the biochemical hallmarks of PRS superactivity. (J. Clin. Invest. 1995. 96:2133-2141.) Key words: gout - point mutation - purine-pyrimidine metabolism, inborn errors • allosteric regulation • ribosephosphate pyrophosphokinase
\end{abstract}

\section{Introduction}

Phosphoribosylpyrophosphate (PRPP) ${ }^{1}$ is a substrate in and an allosteric regulator of the synthesis of purine and pyrimidine

Address correspondence to Michael A. Becker, MC 0930, The University of Chicago Medical Center, 5841 South Maryland Avenue, Chicago, IL 60637. Phone: 312-702-6899; FAX: 312-702-3467.

Received for publication 19 April 1995 and accepted in revised form 5 July 1995.

1. Abbreviations used in this paper: $I_{0.5}$, inhibitory constant; IEF, isoelectric focusing; IPTG, isopropylthiogalactoside; $K_{\mathrm{a}}$, activation constant; Pi, inorganic phosphate; PRPP, 5-phosphoribosyl 1-pyrophosphate; PRPS, phosphoribosylpyrophosphate synthetase gene; PRS, phosphoribosylpyrophosphate synthetase; PVDF, polyvinylidenedifluoride.

J. Clin. Invest.

(c) The American Society for Clinical Investigation, Inc.

$0021-9738 / 95 / 11 / 2133 / 09 \quad \$ 2.00$

Volume 96, November 1995, 2133-2141 nucleotides (1). Synthesis of PRPP from ATP and Rib-5-P is catalyzed by PRPP synthetase (PRS; EC 2.7.6.1) in a reaction requiring inorganic phosphate $(\mathrm{Pi})$ and $\mathrm{Mg}^{2+}$ both as cofactors and as activators(2-4). PRS activity is inhibited by purine, pyrimidine, and pyridine nucleotide products of the multiple pathways of PRPP utilization as well as by the products of the PRS reaction and certain additional phosphorylated compounds $(1,4)$ and synthetic nucleotide analogs $(5,6)$.

PRS superactivity is an $X$ chromosome-linked purine metabolic defect in humans (7), characterized by gout and uric acid overproduction (8) resulting from accelerated synthesis of PRPP and purine nucleotides $(9,10)$. Kinetic defects underlying PRS superactivity include: $(a)$ regulatory defects characterized by purine nucleotide inhibitor-resistance, sometimes associated with increased apparent affinity of PRS for Pi $(9,11-$ $14)$; (b) catalytic defects in which maximal reaction velocity is increased but substrate and activator affinities and inhibitor responsiveness are normal $(15-19) ;(c)$ combined regulatory and catalytic defects $(20,21)$; and $(d)$ increased affinity for the substrate Rib-5-P (22). Although regulatory defects comprise all or a part of the kinetic aberrations resulting in PRS superactivity in only 6 of the nearly 30 affected families studied in detail, infantile or childhood onset and neurodevelopmental impairment accompany hyperuricemia and gout in five of these families $(12,13,20,23)$, but are rare in families with other defects $(17,24)$. Our aims were to define and characterize specific genetic aberrations leading to PRS superactivity with regulatory defects and to apply the resulting information to an understanding of the determinants of expression of PRS activity.

Prior studies $(25,26)$ have identified two X chromosomal loci (PRPS1 and PRPS2) encoding highly homologous PRS cDNAs (PRS1 and PRS2 cDNAs, respectively, which have $81 \%$ nucleotide identity in the 954-bp translated regions) and PRS proteins (95\% predicted amino acid identity) $(27,28)$. Human PRPS genes each encompass $\sim 35-\mathrm{kb}$ of DNA and contain seven exons (Becker, M.A., unpublished data) and proximal 5'-untranscribed sequence consistent with a housekeeping function (29). PRPS1 maps to the interval Xq22$\mathrm{q} 24$ and PRPS2 to Xp22.2-p22.3 (26). Although there are tissue-specific differences in expression of PRS1 and PRS2 transcripts (30) and in the kinetic and physical properties of PRS1 and PRS2 isoforms (31), little is known about the functional significance of these differences in vivo.

Cloning and sequencing of PRS1 and PRS2 cDNAs derived from two unrelated affected hemizygous male children established that the genetic basis of PRS superactivity associated with purine nucleotide inhibitor resistance in some families at least is point mutation in the PRPSI gene (32). We have now extended the analysis of $\mathrm{X}$ chromosome-encoded PRS cDNAs and their respective isoforms to all six families with regulatory defects in PRS activity. In each affected family, a distinctive point mutation in the translated region of the PRPSI gene has 
been identified, and the respective mutant PRS1 isoforms have been expressed in Escherichia coli and compared to recombinant normal PRS1 with regard to enzymatic and physical properties. Our studies show that amino acid substitutions over a fairly wide stretch of PRS1 subunit primary structure result in alteration of the allosteric regulatory properties of the enzyme, notably affecting both purine nucleotide inhibitory and Pi activating mechanisms.

\section{Methods}

Cell lines. Fibroblast strains were initiated and propagated from skin biopsies obtained from four normal individuals and from six unrelated hemizygous male patients with PRS superactivity associated with impaired purine nucleotide inhibitor responsiveness $(9,12,13,20)$, including patients NB and SM whose PRS cDNAs have previously been studied (32). Cultures were grown in monolayer in Eagle's MEM supplemented with $10 \%$ FBS, $2 \mathrm{mM}$ L-glutamine, and nonessential amino acids. Cells were studied when cultures had reached late log phase and were lightly confluent. Cell monolayers were washed twice in serumfree medium before extraction of total RNA for reverse transcription and PRS cDNA amplification. To prepare cell-free extracts for PRS activity assay or protein analysis, cultures were rinsed three times in serum-free medium before treatment with trypsin-EDTA, as described (20).

RNA isolation and PCR amplification of PRS $c D N A$. Total cellular RNA was isolated from 3 to $5 \times 10^{7}$ fibroblasts (33), and PCR amplification of PRS cDNA was accomplished as described (32). Briefly, reverse transcription of RNA into CDNA was primed with a PRS1- or PRS2-specific antisense primer, and a portion of the incubation mixture was diluted and subjected to amplification of PRS cDNAs in PCRs catalyzed by TaqI DNA polymerase (34) and primed with appropriate oligodeoxynucleotide primer pairs corresponding to the respective $5^{\prime}$ and 3 '-untranslated sequences of normal human PRS1 and PRS2 cDNAs (32). By this approach, the entire translated regions of the PRS1 and PRS2 cDNAs were amplified for the initial sequencing and cloning studies described below.

Sequencing. Sequencing of amplified PRS1 and PRS2 cDNAs was carried out directly from PCR mixtures by means of a modification of the dideoxynucleotide chain termination method $(34,35)$. The translated regions of both strands of PRS1 and PRS2 cDNAs were fully sequenced using both the amplification primers and series of PRS consensus sequencing primers that hybridized to PRS1 and PRS2 coding sequences internal to the PCR amplification primers (32). In subsequent sequencing experiments confirming the fidelity and orientation of PRS1 cDNA sequences in the plasmid pSPRBS expression vector, internal PRS sequencing primers as well as appropriate plasmid primer sequences were utilized to sequence both strands of the PRS1 cDNAs, and standard dideoxynucleotide sequencing was used (34).

Expression of normal and mutant PRS1 cDNAs. A standardized method of preparation of PRS1 cDNAs for expression, differing from previous procedures (32), was developed. A portion of each PRS1 PCR amplification mixture, prepared as described above, served as template for a second round of 30 cycles of PCR utilizing a nested pair of primers that incorporated EcoRI (sense strand) and XhoI (antisense strand) restriction sites into the sequence of PRS1 cDNA immediately upstream to the $5^{\prime}$ translation initiation codon and 44-bp downstream of the 3 termination codon, respectively. Primer sequences were: 5'GTTGGAATTCATGCCGAATATCAAAATCTTCAGCGG3' ; and 5'GCTACACTCGAGCAAGCCGGGTC 3 '. Products of the second amplification reactions were digested with EcoRI and XhoI and cloned into the corresponding restriction sites in the multiple cloning region of PSPRBS (32), a previously described plasmid expression vector derivative of pSP72 containing a ribosomal binding site immediately upstream of the EcoRI restriction site and downstream of the T7 phage RNA polymerase promoter (36). Recombinant plasmids bearing normal or mutant human PRS1 cDNA-translated sequences were then used to transform $E$. coli strain $\mathrm{DH} 5 \alpha$, and bacterial colonies surviving growth on ampicillin were screened for the presence of the recombinant vector by restriction analysis and by sequencing of the human PRS1 cDNA translated and adjacent regions.

Once correct orientation and, where appropriate, the presence of the respective mutation in the PRS1 cDNA were verified by dideoxy sequencing, the plasmid was used to transform $E$. coli BL21 (DE3/ pLysS), a strain lysogenized with DE3 phage bearing the T7 RNA polymerase gene under the control of the isopropylthiogalactoside (IPTG)-inducible lac UV5 promoter (37). Bacterial colonies surviving growth on ZB plates (36) containing ampicillin and chloramphenico were incubated in $\mathrm{ZB} / \mathrm{M} 9$ medium (36) in the presence of antibiotics and were grown at $37^{\circ} \mathrm{C}$ to an absorbance of 0.4 at $600 \mathrm{~nm}$ before addition of $0.4 \mathrm{mM}$ IPTG and $2 \mathrm{~h}$ of additional incubation. Bacterial cells were harvested, resuspended in a lysis buffer, and disrupted, all as previously described (31). After centrifugation at $10,000 \times g$ for $10 \mathrm{~min}$, samples of the supernatant layers of the cell extracts were electrophoresed on $12 \%$ SDS-PAGE slab gels (38) and on $4.5 \%$ polyacrylamide-9.2 M urea isoelectric focusing (IEF) gels (39) and were assayed for PRS activities and kinetic properties. In certain instances, recombinant PRSIs were purified to near homogeneity as previously described (31).

SDS-PAGE gels were analyzed by staining with $0.1 \%$ Coomassie brilliant blue, or, alternatively, subjected to electrotransfer of proteins to polyvinylidenedifluoride (PVDF) membranes for immunoblot analysis (31) utilizing the IgG fraction of the serum of rabbits immunized with highly purified human erythrocyte PRS (15). IEF gels contained 0.6 $\mathrm{ml}$ of ampholytes ( $0.1 \mathrm{ml} \mathrm{pH} \mathrm{5-8;0.1} \mathrm{ml} \mathrm{pH} \mathrm{6-7;} 0.4 \mathrm{ml} \mathrm{pH} \mathrm{3.5-10)}$ per $10 \mathrm{ml}$ gel solution. Upon completion of electrophoresis, portions of IEF gels were subjected to staining with Coomassie blue, immunoblotting with rabbit PRS antiserum, and fractionation for verification of the $\mathrm{pH}$ gradient of the gel.

PRS assays were performed by a two-step procedure previously described (15), except that the $\mathrm{pH}$ of the first step reaction mixture was adjusted to 8.0 and dilutions of the bacterial extract supernatant layers were in lysis buffer containing $50 \mathrm{mM}$ Tris- $\mathrm{HCl}$ ( $\mathrm{pH} 8.0$ ), an appropriate sodium Pi concentration, and $1 \mathrm{mg} / \mathrm{ml}$ BSA (32). Recombinant human PRS1s in bacterial cell extracts were analyzed with regard to affinity for substrates (15), Pi activation (13), and inhibition by ADP and GDP $(32,40)$. In all studies of the kinetics of recombinant PRS1s, controls for bacterial PRS activities were included, utilizing extracts of bacterial cells transformed with pSPRBS devoid of a human PRS1 cDNA. Substrate affinity and nucleotide inhibitor studies were carried out at $1.0 \mathrm{mM} \mathrm{Pi}$, a concentration at which $E$. coli PRS activity is virtually undetectable (32). At each $\mathrm{Pi}$ concentration tested in the $\mathrm{Pi}$ activation studies, bacterial PRS activity was $<2 \%$ of the total PRS activity measured in bacterial cells transformed with a vector containing a human PRS1 cDNA. Protein concentrations of cell extracts were measured by the method of Lowry et al. (41), with BSA as standard. Specific activities of PRS are expressed as units (micromoles of PRPP formed per min) per milligram of protein.

\section{Results}

PRS1 and PRS2 cDNA sequences. The entire translated sequences of PRS1 and PRS2 cDNAs from normal individuals and each of the six unrelated male patients were directly ascertained from PCR pools after reverse transcription of total fibroblast RNA and 30 cycles of cDNA amplification primed by appropriate PRS1- and PRS2-specific amplification primers. All normal and patient PRS2 cDNA sequences were identical throughout the translated region. However, the PRS1 cDNA sequence derived from each of the six affected patients differed from the others and from normal PRS1 cDNA sequence by a single base substitution, predicting replacement of the corresponding amino acid residue of normal PRS1 with a different 
Table I. Mutations in PRSI in Patients with PRS Superactivity and Purine Nucleotide Inhibitor Resistance

\begin{tabular}{llll}
\hline Patient & $\begin{array}{c}\text { Base } \\
\text { Substitution }\end{array}$ & \multicolumn{1}{c}{$\begin{array}{c}\text { Type of } \\
\text { mutation }\end{array}$} & $\begin{array}{c}\text { Deduced amino } \\
\text { acid replacement }\end{array}$ \\
\hline OG & G154 $\rightarrow$ C & Transversion & Asp51 $\rightarrow$ His \\
NB & A341 $\rightarrow$ G & Transition & Asn113 $\rightarrow$ Ser \\
RD & C385 $\rightarrow$ A & Transversion & Leu128 $\rightarrow$ Ile \\
SM & G547 $\rightarrow$ C & Transversion & Asp182 $\rightarrow$ His \\
AL & C569 $\rightarrow$ T & Transition & Ala189 $\rightarrow$ Val \\
VRG & C579 $\rightarrow$ G & Transversion & His192 $\rightarrow$ Gln
\end{tabular}

As described in Methods, the entire translated regions of PRS1 cDNAs, prepared by PCR amplification from the total RNA of normal and patient fibroblasts, were sequenced and compared. A unique base substitution relative to normal PRS1 cDNA was demonstrated in each patient-derived PRS1 cDNA on at least two occasions. The substitutions indicated for SM and NB PRS1 cDNAs agree with those previously described (32). Nucleotides are numbered relative to the A residue of the ATG initiation codon. In mature PRS1, $\mathrm{NH}_{2}$-terminal Met has been removed. As a consequence, amino acid residues are numbered relative to the $\mathrm{NH}_{2}$-terminal Pro residue corresponding to nucleotides 4-6.

amino acid residue (Table I). Each altered PRS1 cDNA sequence was confirmed on both strands of the respective cDNA. RNA isolation, reverse transcription, amplification, and sequencing of PRS1 and PRS2 cDNAs derived from the fibroblasts of each patient and control were carried out on at least two occasions, and the identical base substitution was identified in the respective patient-derived PRS1 cDNA on all occasions. In addition to confirmation of an $A$ to $G$ transition at nucleotide position 341 in patient NB PRS1 cDNA and a $G$ to $C$ transversion at nucleotide position 547 in patient SM PRS1 cDNA (32), new base substitutions (three transversions and one transition) were demonstrated in the PRS1 cDNAs of the other four patients by this approach.

Open reading frames of $954 \mathrm{bp}$ from the ATG translation initiation codon to the TTA codon preceding the chain termination TAA signal were demonstrated for all normal- and patientderived PRS1 cDNAs. Specifically, no deletions, additions, frameshifts or premature chain termination signals were detected, so that PRS1 cDNA sequencing predicted mature polypeptides of 317 amino acid residues $\left(\mathrm{NH}_{2}\right.$-terminal Met cleaved) and molecular masses of $\sim 34.7 \mathrm{kD}$ for all mature PRS1s.

The single amino acid residue changes in PRS1 primary structure predicted from the nucleobase substitutions in PRS1 cDNAs (Table I) ranged from conservative replacements (for example, Ile for Leu in patient RD PRS1) to those resulting in the substitution of a basic residue (His) for an acidic amino acid (Asp) in both patients SM and OG PRS1s. Moreover, the predicted amino acid substitutions encompassed a region nearly half the length of the 317 amino acid sequence of the PRS1 polypeptide, extending from the Asp51His substitution in patient OG PRS1 to the His192Gln replacement predicted for VRG PRS1.

Expression of recombinant normal and patient-derived PRS1 isoforms. To establish the relationship between the single base substitutions identified in the patient-derived PRS1 cDNAs and the aberrant kinetic properties of the respective PRSs previously detected in cultured cells from the affected individuals, normal and patient PRS1 cDNAs were cloned into pSPRBS. After the sequences of both strands of the cloned cDNAs were confirmed, the plasmid and recombinant derivatives were used to transform E. coli BL 21 (DE3/pLysS) (Recombinant plasmids bearing human PRS1 cDNA translated sequences are denoted pSPRS1 with a suffix indicating the human PRS1 cDNA incorporated.) Under conditions of induction with IPTG, normal and mutant PRS1 cDNAs were expressed at high levels in the bacterial strain, with recombinant human PRS1 representing 3 to $12 \%$ of total bacterial cell extract protein as assessed by PRS1 specific activity (31) and the results of SDS-PAGE and immunoblot analyses (Fig. 1). In contrast to extracts of bacterial cells transformed with pSPRBS alone, extracts of cells transformed with vector containing a normal or patient-derived PRS1 cDNA (except patient OG PRS1 cDNA) showed a prominent protein corresponding to $34.5 \mathrm{kD}$ on SDS-PAGE gels stained with Coomassie blue (Fig. $1 A$ ). The identity of this protein as recombinant human PRS1 was confirmed by immunoblotting, which showed that the IgG fraction of serum from rabbits immunized to purified human PRS reacted with the 34.5$\mathrm{kD}$ protein (Fig. $1 B$ ), but unimmunized rabbit IgG did not (not shown). The findings for recombinant PRS1 are in good agreement with the molecular mass of the normal human PRS1 isoform subunit predicted from the cDNA sequence $(34.7 \mathrm{kD}$ ) (27) and estimated for the human erythrocyte PRS subunit by physical methods (34.5 kD) (3).

Expression of one of the six mutant PRS1 cDNAs (patient OG PRS1 cDNA) consistently resulted in production of a recombinant protein displaying PRS1 enzymatic activity and immunoreactivity but with reduced apparent molecular mass ( $\sim 33.8 \mathrm{kD}$ ) (Fig. 1) despite the normal length of patient OG PRS1 cDNA. The apparently smaller recombinant patient OG PRS1 was also identifiable when patient OG PRS1 cDNA-transformed $E$. coli cells were pelleted immediately after IPTG induction and were extracted with boiling SDS-PAGE sample buffer and when patient OG fibroblasts were subjected to the same treatment. These findings could reflect either aberrant mobility of an intact PRS1 isoform under denaturing conditions or a proteolytic cleavage event not shared with normal and other mutant PRS1 isoforms. In either case, the point mutation resulting in a substitution of His for Asp at PRS1 residue 51 seems likely to be a key determinant of this finding. When patient OG PRS1 was purified to $>90 \%$ homogeneity and was subjected to gas phase protein sequencing, the $\mathrm{NH}_{2}$-terminal 20 residues of patient OG PRS1 were identical to those of normal PRS1 $(27,31)$, dismissing $\mathrm{NH}_{2}$-terminal cleavage as the basis of the reduced apparent molecular mass of patient OG PRS1.

Immunoblot analysis was applied to recombinant PRS1s in bacterial cell expression extracts or to purified recombinant PRS1 preparations after electrophoresis on polyacrylamide-urea IEF gels (Fig. 2). In this system, recombinant normal PRS1 was identifiable as a doublet, the two bands showing pIs of $\sim 6.8$ (rat and human PRS1s are identical in amino acid sequence $(27,42)$; nevertheless, different pIs have been reported for recombinant rat (42) and human (31) PRS1s. Both values are substantially lower than that determined for the recombinant normal PRS1 in this study, which, in turn, agrees closely with the pI of PRS1 (6.8), predicted from the amino acid sequence.) A doublet of identical mobility was detectable in crude extracts of cultured normal human fibroblasts. In the latter case, however, PRS1 bands were accompanied by additional bands corresponding in mobility to those of purified recombinant normal 

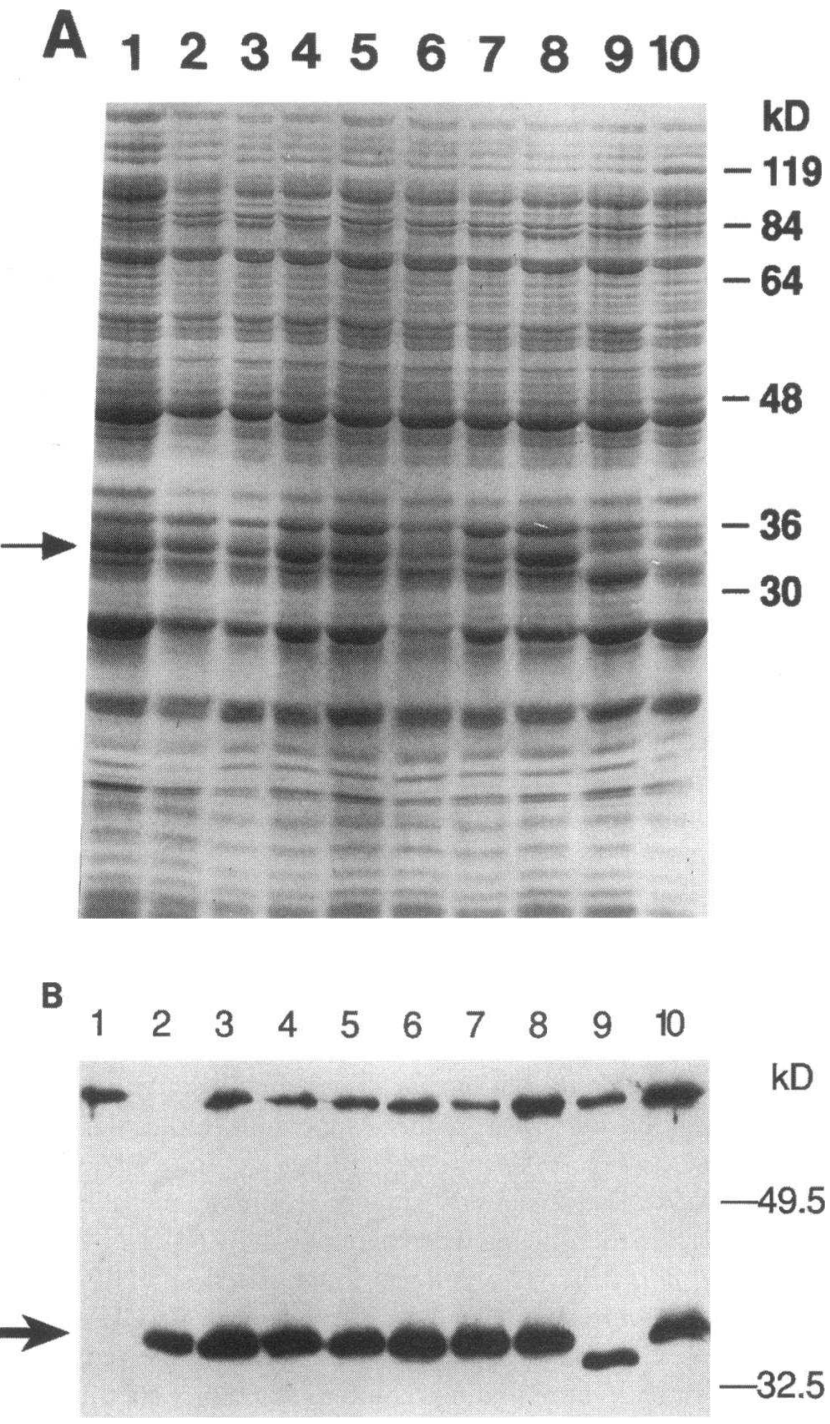

Figure 1. SDS-PAGE analysis by protein staining $(A)$ and immunoblotting $(B)$ of supernatant fractions of crude extracts from $E$. coli BL21 (DE3/pLysS) transformed with the indicated constructs and induced for $2 \mathrm{~h}$ with $0.4 \mathrm{mM}$ IPTG, as described in Methods. Locations of molecular mass standards are indicated. $(A)$ Lane 1, pSPRS1-N1 (normal ); lane 2, pSPRS1-SM1; lane 3, pSPRS1-NB1; lane 4, pSPRS1-N2 (normal); lane 5, pSPRS1-HB1 (PRS1 cDNA derived from a patient with catalytic PRS superactivity); lane 6, pSPRS1-VRG1; lane 7, pSPRS1-RD1; lane 8, pSPRS1-AL1; lane 9, pSPRS1-OG1; lane 10, pSPRBS. Each lane contained $30 \mu \mathrm{g}$ of crude extract protein, and proteins were stained with $0.1 \%$ Coomassie brilliant blue. The arrow indicates location of a protein band at $34.5 \mathrm{kD}$, appearing in extracts of pSPRS1-transformed cells (lanes 1-8) but not in extracts of pSPRBStransformed cells. Although the $34.5-\mathrm{kD}$ band is not seen in lane 9, a band of with slightly increased mobility and greater intensity than in corresponding regions of the other lanes is present at $\sim 33.8 \mathrm{kD}$. (B) Lane 1, pSPRBS; lane 2, purified recombinant normal PRS1 (20 ng); lane 3, pSPRS1-N1; lane 4, pSPRS1-SM1; lane 5, pSPRS1-NB1; lane 6, pSPRS1-AL1; lane 7, pSPRS1-RD1; lane 8, pSPRS1-VRG1; lane 9, pSPRS1-OG1; lane 10, pSPRS1-HB1. Lanes other than lane 2 contained 1-3 $\mu \mathrm{g}$ of crude extract protein. After electrophoretic separation, proteins were electroblotted onto a PVDF membrane, which was incubated with rabbit anti-human PRS IgG (15) and then horseradish peroxidaselinked donkey anti-rabbit Ig (Amersham Life Science). The membrane was washed, immersed in ECL Western Blot detection reagent (Amersham Life Science, Arlington Heights, IL) and exposed to X-ray film for $30 \mathrm{~s}$. A specific immunoreactive band $(34.5 \mathrm{kD})$ with electrophoretic
123456789 Figure 2. Immunoblot

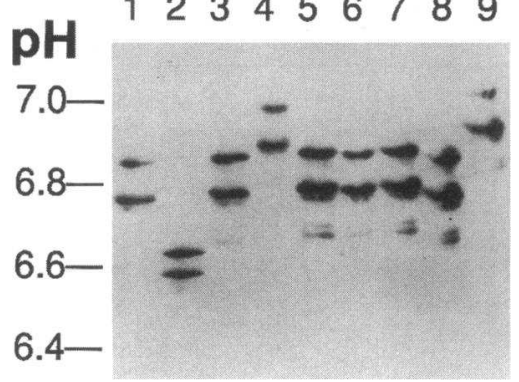

analysis of supernatant fractions of crude extracts from $E$. coli BL21 (DE3/pLysS) transformed with the indicated plasmid constructs and induced for 2 $\mathrm{h}$ with $0.4 \mathrm{mM}$ IPTG, as described in Methods.

After electrophoresis of cell extract samples

(1.5-4.0 $\mu \mathrm{g}$ of protein) on a polyacrylamide-urea IEF gel and electrotransfer of protein to a PVDF membrane, immunoblotting was carried out, as described in Fig. 1 except that exposure to x-ray film was for 5 s. Lane 1, purified recombinant PRS1 (60 ng); lane 2, purified recombinant PRS2 (60 ng) (34); lane 3, pSPRS1-N1; lane 4, pSPRS1-SM1; lane 5, pSPRS1-NB1; lane 6, pSPRS1-AL1; lane 7, pSPRS1-RD1; lane 8, pSPRS1-VRG1; lane 9, pSPRS1-OG1. Normal PRS1 migrates as a doublet of immunoreactive bands with pls near 6.8. Additional weakly immunoreactive bands that migrate with more acidic pIs are visible in some lanes but are detectable in all extracts of cells transformed with pSPRS1-containing vectors when sample protein concentration is increased. $\mathrm{pH}$ values corresponding to regions of the IEF gel after electrophoresis are indicated.

human PRS2 with which the PRS antibodies cross react (31). Although recombinant normal, NB, AL, and RD PRS1s showed bands of identical mobility on isoelectric focusing gels, altered mobility was shown by the PRS1s from the other three affected patients (Fig. 2). Both recombinant PRS1 bands from patients SM and OG showed reduced (more basic) isoelectric mobility, with the OG PRS1 bands retarded slightly more than those of SM PRS1. These variant PRS1s have the same amino acid substitution (His for Asp) at different residues, so that the differential effect of this substitution on the isoelectric points of the respective PRS1s may be a consequence of the mechanism responsible for the reduced apparent molecular mass of $O G$ PRS1. In addition, recombinant VRG PRS1 bands showed slightly increased mobility on isoelectric focusing gel indicative of a more acidic pI for this variant PRS1, consistent with the replacement of His by Gln at amino acid residue 192 .

Kinetic properties of recombinant mutant PRS1s. Kinetic features of the PRS reaction catalyzed by recombinant PRS1s in bacterial cell expression extracts are summarized in Tables II and III. Michaelis constants for the substrates Rib-5-P and MgATP were similar for all recombinant normal and mutant PRS1s (Table II), and double reciprocal plots for substrate saturation were linear. In contrast, all patient-derived recombinant PRS1s were resistant to inhibition of activity by ADP (Fig. $3 A$ ) and, especially, by GDP (Fig. $3 B$ ), as determined by measurement of inhibitory constants $I_{0.5}$ (Table III). There

mobility of purified recombinant normal PRS1 (lane 2) is seen in all lanes (except lane 9) corresponding to extracts of cells transformed with pSPRS1-containing vectors. No specific immunoreactive band is seen in the extract of pSPRBS-transformed cells (lane 1). A single immunoreactive band $(33.8 \mathrm{kD})$ is seen in lane 9 , representing an extract of cells transformed with pSPRS1-OG1. In an identical immunoblotting procedure in which normal (unimmunized) rabbit IgG replaced rabbit anti-human PRS IgG, no specific immunoreactive bands were seen. 
Table II. Kinetic Constants for the PRS1 Reaction Catalyzed by Normal and Mutant rPRS1s

\begin{tabular}{|c|c|c|c|c|}
\hline \multirow{2}{*}{$\begin{array}{c}\text { Recombinant } \\
\text { PRS1 }\end{array}$} & \multicolumn{2}{|c|}{$K_{\mathrm{m}}$ for: } & \multicolumn{2}{|c|}{ Activation constants for $\mathrm{Pi}$} \\
\hline & ATP & Rib-5-P & $K_{\mathrm{a}}$ & $\mathrm{n}_{\mathrm{H}}^{*}$ \\
\hline & \multicolumn{2}{|c|}{$\mu M$} & \multicolumn{2}{|c|}{$m M$} \\
\hline Normal $^{\ddagger}$ & $23 \pm 3$ & $54 \pm 5$ & $0.88 \pm 0.1$ & $3.1 \pm 0.3$ \\
\hline OG & 20 & 52 & 0.26 & 2.3 \\
\hline NB & 28 & 56 & 0.32 & 2.1 \\
\hline RD & 26 & 49 & 0.15 & 1.7 \\
\hline SM & 19 & 51 & 0.14 & 2.1 \\
\hline $\mathrm{AL}$ & 22 & 55 & 0.13 & 2.1 \\
\hline VRG & 21 & 50 & $<0.10^{\S}$ & - \\
\hline
\end{tabular}

Normal and mutant rPRS1s in bacterial expression extracts were diluted in a stabilization buffer at $\mathrm{pH} 8.0$, containing: $50 \mathrm{mM}$ Tris- $\mathrm{HCl} ; 0.3$ mM ATP; 1 mM DTT; 6 mM MgCl $; 1$ mM EDTA; 0.1 mM PMSF; and $1 \mathrm{mg} / \mathrm{ml} \mathrm{BSA}$. When the $K_{\mathrm{m}}$ for ATP was studied, ATP concentration in the stabilization buffer was $0.03 \mathrm{mM}$, and final ATP concentration was varied in the reaction mixture from 7.5 to $100 \mu \mathrm{M}$, with Rib-5-P concentration at $350 \mu \mathrm{M}$. When the $K_{\mathrm{m}}$ for Rib-5-P was studied, Rib5-P concentration was varied from 0 to $200 \mu \mathrm{M}$, with ATP concentration at $500 \mu \mathrm{M}$. In both studies, Pi concentration was $1.0 \mathrm{mM}$. Pi activation was tested over a range of Pi concentrations from 0.025 to $8.0 \mathrm{mM}$, with ATP and Rib-5-P concentrations of 500 and $350 \mu \mathrm{M}$, respectively. * Hill coefficient; ${ }^{\ddagger}$ Values for normal rPRS1 are the average \pm 1 SD of three determinations each for normal rPRS1s from three normal individuals. All other values represent the means of at least three determinations on separate occasions, with agreement to within 15 percent. ${ }^{8} K_{\mathrm{a}}$ value of VRG rPRS1 for Pi is provisional, because only one point below the $K_{\mathrm{a}}$ could be measured. rPRS1, recombinant PRS1.

Table III. Inhibition of Normal and Mutant rPRS1 and Fibroblast Total PRS Activities by ADP and GDP

\begin{tabular}{lrrrrr}
\hline & \multicolumn{5}{c}{$I_{0.5}$ for: } \\
\cline { 2 - 3 } $\begin{array}{l}\text { Source } \\
\text { of PRS } \\
\text { activity }\end{array}$ & \multicolumn{2}{c}{ ADP } & & \multicolumn{2}{c}{ GDP } \\
\cline { 2 - 3 } \cline { 5 - 6 } & rPRS1 & Fibroblast PRS & rPRS1 & Fibroblast PRS \\
\hline & & & $\mu M$ & & \\
Normal & 21 & 31 & & 52 & 74 \\
OG & 75 & 58 & & 225 & 161 \\
NB & 78 & 46 & & 790 & 230 \\
RD & 128 & 91 & $>1,000$ & 267 \\
SM & 150 & 92 & & 810 & 251 \\
AL & 69 & 52 & 565 & 222 \\
VRG & 141 & 106 & 770 & 204 \\
& & &
\end{tabular}

Normal and mutant rPRS1s in bacterial expression extracts were diluted in stabilization buffer at $\mathrm{pH} 8.0$ (Table II legend) containing sufficient $K P_{i}(\mathrm{pH} 8.0)$ to give a final $\mathrm{Pi}$ concentration of $1.0 \mathrm{mM}$ in the reaction mix. Concentrations of ATP and Rib-5-P were 500 and $350 \mu \mathrm{M}$, respectively and the ranges of ADP and GDP concentrations in the assay were $0-500 \mu \mathrm{M}$ and $0-1,000 \mu \mathrm{M}$, respectively. Chromotographed fibroblast extracts, prepared as previously described (20), were also assayed at pH 8.0 in $1.0 \mathrm{mM}$ Pi with identical ATP and Rib-5-P concentrations. Ranges of ADP and GDP concentrations in the assay were $0-250 \mu \mathrm{M}$ and $0-500 \mu \mathrm{M}$, respectively. All values are the means of at least two experiments, with agreement to within 15 percent.


Figure 3. Inhibition of recombinant normal $(\mathrm{O}), \mathrm{OG}(\bullet), \mathrm{RD}(\square), \mathrm{AL}$ $(\Delta)$, and VRG ( $\nabla)$ PRS1s by ADP $(A)$ and GDP $(B)$. Supernatant fractions of transformed $E$. coli BL21 (DE3/pLysS) extracts were assayed for PRS activity at $1.0 \mathrm{mM}$ Pi and the indicated concentrations of the respective inhibitors, as described in Table III. Specific activities ( $\mathrm{U} / \mathrm{mg}$ of protein) of the recombinant PRS1s in the absence of ADP and GDP were: normal, 0.51; OG, 0.67; RD, 0.48; AL, 0.69; VRG, 0.39 . Specific activity of bacterial PRS at $1.0 \mathrm{mM} \mathrm{Pi}$, determined in extracts of cells transformed with pSPRBS, was $0.003 \mathrm{U} / \mathrm{mg}$. Purified recombinant normal PRS1 had a specific activity of $13.1 \mathrm{U} / \mathrm{mg}$ at 1 $\mathrm{mM}$ Pi. Data for recombinant SM and RD PRS1s have previously been presented (32).

were, in addition, differences in the patterns and magnitudes of purine nucleoside diphosphate inhibitor resistance among the mutant enzymes. For example, NB and AL PRS1s showed relatively slight resistance to inhibition by ADP but were substantially more resistant to GDP. Recombinant OG PRS1 was also among the least resistant to ADP inhibition and was considerably less resistant to GDP than the other recombinant mutant PRS1s. RD, SM, and VRG PRS1s exhibited prominent resistance to both ADP and GDP. Each recombinant PRS1 was compared with fibroblast PRS from the corresponding patient with respect to inhibition of enzyme activity by the purine nucleotides (Table III). Overall, the pattern of inhibitor responsiveness was similar for recombinant PRS1 and fibroblast PRS activities from each patient. In all instances, however, the recombinant mutant PRS1 isoform showed greater ADP and GDP 

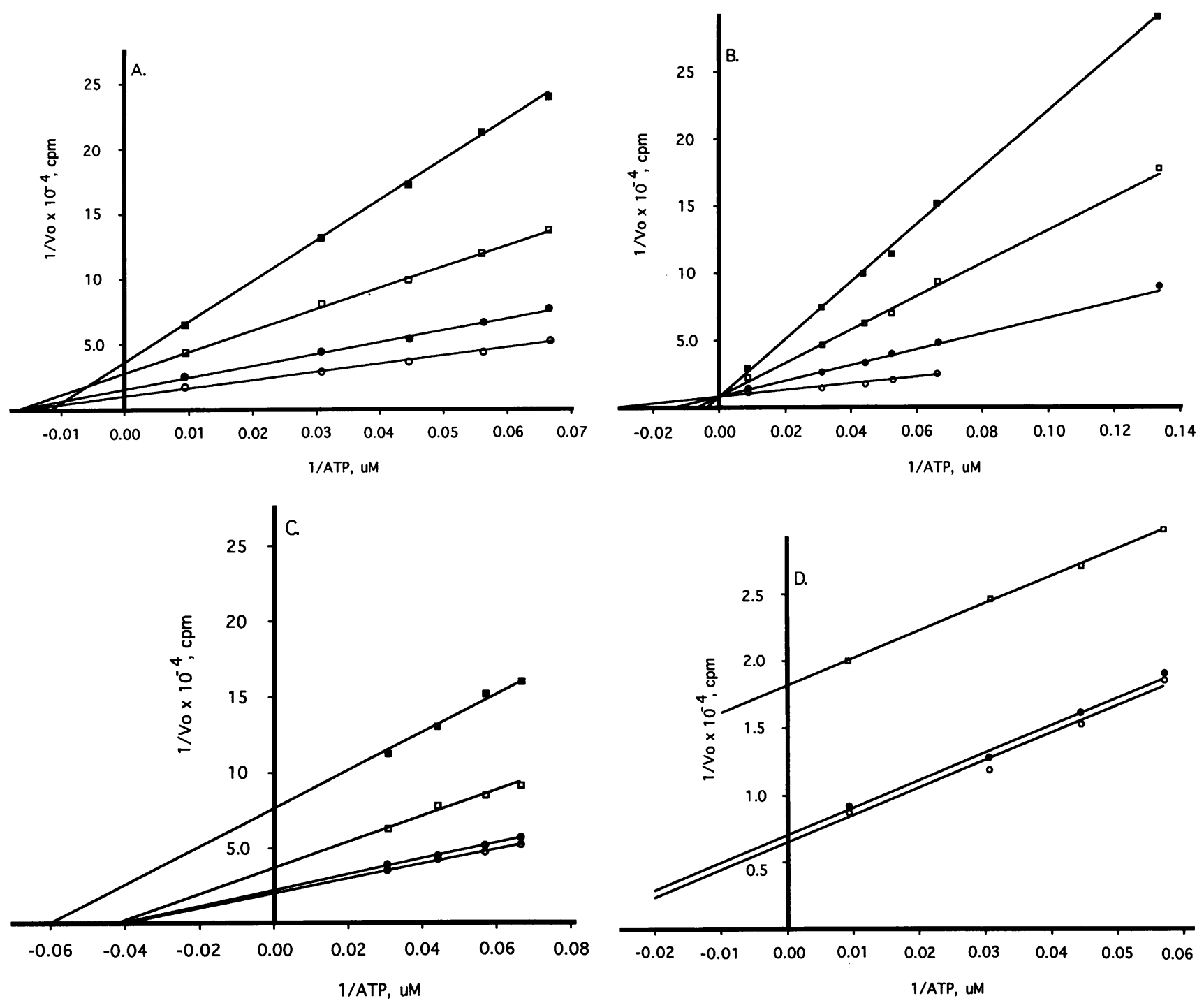

Figure 4. Double reciprocal plots of the inhibition of purified recombinant PRS1s by ADP ( $A$ and $B$ ) and GDP ( $C$ and $D$ ) at varying ATP concentrations. (A) Recombinant normal PRS1. ADP concentrations were: $\mathrm{O},(\mathrm{O}) ; 10 \mu \mathrm{M},(\bullet) ; 20 \mu \mathrm{M},(\square)$; and $30 \mu \mathrm{M},(\bullet)$. (B) Recombinant OG PRS1. ADP concentrations were: $\mathrm{O},(\mathrm{O}) ; 10 \mu \mathrm{M}(\bullet) ; 30 \mu \mathrm{M},(\square) ; 50 \mu \mathrm{M},(\bullet)$. (C) Recombinant normal PRS1. GDP concentrations were: $\mathrm{O},(\mathrm{O}) ; 10 \mu \mathrm{M},(\bullet) ; 50 \mu \mathrm{M},(\square) ; 100 \mu \mathrm{M},(\bullet)$. (D) Recombinant OG PRS1. GDP concentrations were: O, (O); $100 \mu \mathrm{M},(\bullet) ; 250 \mu \mathrm{M}(\square) . K_{\mathrm{i}}$ slope values, calculated from secondary plots of the slopes determined in panels $A$ and $B$ versus ADP concentrations, were 3 and $6 \mu \mathrm{M}$ for normal and OG PRS1s, respectively. The corresponding $K_{\mathrm{i}}$ slope value for purified SM PRS1 was $6 \mu \mathrm{M}$. The $K_{\mathrm{i}}$ intercept value for normal PRS1 was $7 \mu \mathrm{M}$.

inhibition resistance than the corresponding fibroblast PRS. This finding most likely reflects coexpression in fibroblasts of PRS1 and PRS2 (30), with total PRS activity representing a composite of the independent activities of PRS1 and the normally less purine nucleotide-sensitive PRS2 isoform (31). The demonstration that, for each family, a single base substitution in PRS1 cDNA results in a recombinant PRS1 with enzymatic properties recapitulating those found in cells from the respective affected individual reinforces the view that mutation in a region of PRPS1 encoding PRS1 structure provides the genetic basis for PRS superactivity associated with purine nucleotide inhibitor resistance.

The mechanisms of ADP and GDP inhibition of human PRS1s were studied at $1.0 \mathrm{mM} \mathrm{Pi}$ in purified preparations of the recombinant normal, OG (Fig. 4), and SM (data not shown) isoforms. These mutant PRS1s were chosen as representatives of the less and more severely inhibition-impaired recombinant isoforms, respectively. ADP inhibition of normal PRS1 was kinetically complex, appearing to involve both noncompetitive and competitive (with respect to ATP) mechanisms (Fig. $4 A$ ). In contrast, inhibition of purified OG (Fig. $4 B$ ) and SM PRS1s by ADP was best explained by a purely competitive mechanism. The $K_{\mathrm{i}}$ slopes for ADP competitive inhibition of the two recombinant mutant PRS1s were indistinguishable and were not substantially different from the estimated $K_{\mathrm{i}}$ slopes and $K_{\mathrm{i}}$ intercepts for purified normal PRS1 (Fig. 4 legend). GDP inhibition of normal PRS1 (Fig. $4 C$ ) was also complex, displaying features suggesting a strong mechanism of noncompetitive inhibition as well as a weaker component of uncompetitive inhibition. Only the latter mechanism was apparent, though reduced in magnitude, when GDP inhibition of the recombinant mutant enzymes was studied (Fig. $4 D$ ); in the case of the mutant PRS1s, the 


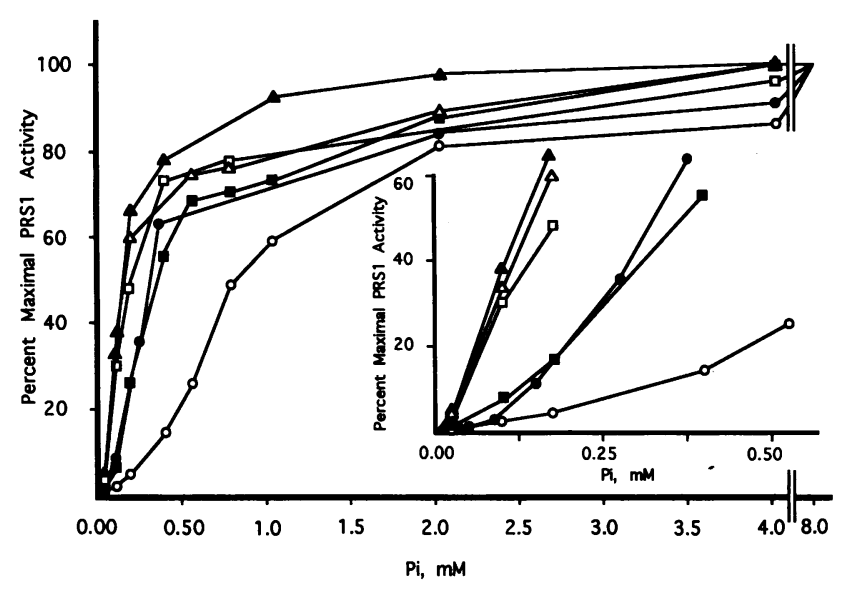

Figure 5. Pi activation of recombinant human PRS1s in supernatant fractions of crude extracts from $E$. coli BL21 (DE3/pLysS) transformed with normal and mutant pSPRS1 vectors and assayed as described in Table II. PRS activities are expressed relative to maximal activities at $8.0 \mathrm{mM}$ Pi. At all Pi concentrations tested, bacterial PRS activity in extracts of cells transformed with pSPRBS were $<2 \%$ those measured in extracts expressing human PRS1s. Inset provides an expanded scale to display contours of Pi activation curves more clearly. Normal PRS1,

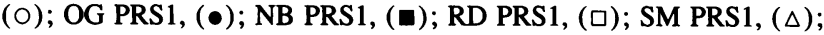
AL PRS1 ( $\triangle$ ). VRG PRS1 is not shown.

noncompetitive mechanism of inhibition by GDP was not detectable.

$\mathrm{Pi}$ activation of recombinant normal PRS1s was sigmoidal, with apparent activation constants $\left(K_{\mathrm{a}}\right)$ ranging from 0.8 to 1.0 $\mathrm{mM}$ (Table II). In contrast, half-activation of all recombinant mutant PRS1s by Pi occurred at substantially lower concentrations. Recombinant NB and OG PRS1s, like normal PRS1s, were clearly activated in a sigmoidal fashion, with apparent $K_{\mathrm{a}}$ of 0.32 and $0.26 \mathrm{mM}$, respectively (Fig. 5). Sigmoidal Pi activation of recombinant RD, SM, AL, and VRG PRS1s was less readily apparent in the range of Pi that could be tested, but double-reciprocal plots of $\mathrm{Pi}$ activation for these enzymes (Fig. 5 inset) displayed cooperative activation with apparent $K_{\mathrm{a}}$ of $0.15,0.14,0.13$, and $<0.10 \mathrm{mM}$, respectively. Increased sensitivity to Pi activation shown by mutant PRS1s was accompanied by a decrease in the apparent degree of cooperativity in response of mutant isoforms to $\mathrm{Pi}$, as assessed by measurement of Hill coefficients (43) (Table II). In general, the increased sensitivity of a mutant PRS1 isoform to activation by Pi did not closely parallel the degree of nucleotide inhibitor resistance displayed by that mutant isoform.

\section{Discussion}

The present work expands the analysis of mutations and molecular mechanisms underlying PRS superactivity associated with purine nucleotide inhibition resistance to all four previously described affected families $(9,12,13,20)$ and to two additional families. Cloning and sequencing of PRS1 cDNAs derived from affected male members of these families demonstrate a distinctive point mutation in the $\mathrm{X}$ chromosome-linked PRPS1 gene in each family. Expression of the mutant PRS1 cDNAs in E. coli establishes that the functional consequence of each mutation is a purine nucleotide inhibitor-resistant PRS1 capable of mediating the accelerated PRPP and purine synthesis that constitute the biochemical hallmarks of PRS superactivity in the cells of affected individuals $(9,10)$. Whether or not other PRS isoforms are coexpressed, the aberrant PRS1 isoforms identified here function as dominant negative mutations with regard to the allosteric regulation of total PRS activity and directly account for both the X-linked pattern of inheritance of this disorder $(7,44)$ and the metabolic aspects of the clinical phenotype: hyperuricemia and gout with uric acid overproduction $(1,8)$. On the other hand, the present studies provide no comparably compelling mechanistic explanation for the neurodevelopmental manifestations of PRS superactivity, which, together with early childhood-onset metabolic consequences, are expressed in all of the patients studied here except for OG. Although recombinant OG PRS1 displays the least prominent overall purine nucleotide feedback-resistance among the mutant enzymes studied, formulation of a direct relationship between the magnitude of PRS overactivity with increased nucleotide synthesis on the one hand and the severity of phenotypic expression on the other is clearly premature. For example, maximal erythrocyte PRS activities in all male patients with regulatory defects in PRS except OG (8) are markedly deficient (8, 12, 20, Becker, M.A., unpublished data), probably as a result of increased enzyme lability (20). This raises an alternative possibility, namely that neurological and developmental consequences of PRPS1 mutations may reflect PRS1 deficiency in tissues mainly populated by postmitotic cells with little PRS2 expression (30).

Point mutations dispersed over a major portion of the PRS1 subunit result in diminished responsiveness to ADP and GDP, indicating a complex and probably extensive allosteric nucleotide inhibitory mechanism and implying that most or all of these mutations alter the transmission of allosteric effects to the active site of PRS1 rather than the primary structure of nucleoside diphosphate-binding residues in the allosteric site. In fact, the identity of nucleotide binding residues in neither the active (presumably ATP/ADP binding) site nor the allosteric site of PRS1 are known. Of the three regions of PRS1 sequence proposed as potential ATP binding segments (45), only the His 192 residue is altered among the mutant PRS1s demonstrated here (patient VRG), but the $K_{\mathrm{m}}$ for ATP is normal for VRG PRS1. The results of a recent study using chimeric rat PRS isozymes (46) demonstrated a critical role for the Lys-4 residue of PRS1 in the sensitivity of the enzyme to GDP and, to a lesser degree, ADP inhibition. Additional sites of sequence divergence between PRS1 and PRS2 also contributed to the differential sensitivities of the isozymes to nucleotide inhibitors (46), confirming both the complexity of the allosteric inhibitory mechanism and the likelihood that GDP and ADP inhibit at least in part by a common molecular process. Each of the mutations identified in the current study occurs at an amino acid residue identical in normal human PRS1 and PRS2 isozymes.

The allosteric regulation of PRS1 activity is complex. Inhibition of purified recombinant normal human PRS1 by ADP appears to involve noncompetitive as well as competitive mechanisms (Fig. $4 A$ ) (40), but only a competitive mechanism of ADP inhibition is demonstrable for recombinant OG (Fig. $4 B$ ) and SM (40) PRS1s. Moreover, the apparent potency of ADP competitive inhibition is comparable for recombinant normal and mutant enzymes when $K_{\mathrm{i}}$ slope values are determined even though SM and OG PRS1s are clearly resistant to inhibition (increased $I_{0.5}$ ) by both ADP and the "noncompetitive" inhibitor GDP (4) in the presence of saturating concentrations of ATP (Table III). These findings are best reconciled by the 
view that point mutations in PRS1 resulting in ADP and GDP resistance disrupt at least a major noncompetitive component of the allosteric nucleotide inhibitory mechanism $(32,40)$ without altering the ATP substrate binding (catalytic) region, the presumed site of ADP competitive inhibition (4). This view is reinforced by the observation that the $K_{\mathrm{m}}$ values for ATP are essentially the same for all six mutant enzymes as for the normal PRS1. The fact that cells with mutations abolishing the allosteric mechanism of ADP (and GDP) inhibition overproduce PRPP and purine nucleotides despite an intact competitive ADP inhibition process makes it unlikely that the latter mechanism is important in exerting control on PRS1 activity under physiological conditions in which ATP concentrations are substantially saturating for the enzyme. The complex kinetics encountered in the study of GDP inhibition of recombinant PRS1s (Fig. 4, $C$ and $D$ ) suggest, however, that the allosteric nucleotide inhibitory process may involve mechanisms that, while largely noncompetitive, still have some dependence on ATP concentration.

For each of the six recombinant mutant PRS1s studied, decreased sensitivity to purine nucleotide inhibitors is also associated with increased sensitivity to allosteric activation by $\mathrm{Pi}$ (Table II). All of the mutant PRS1s are activated at Pi concentrations lower than those activating normal PRS1 and show reduced cooperativity in response to this effector. Nevertheless, no consistent quantitative correlation between the effects of individual mutations on $I_{0.5}$ for ADP and GDP and $K_{\mathrm{a}}$ for Pi is apparent. For example, recombinant SM and OG PRS1s differed much more prominently in the magnitudes of their purine nucleotide inhibitor resistance (Table III) than in their respective sensitivities to Pi activation (Table II). Although delineation of the physical determinants of the effects of purine nucleotide inhibitors and Pi on PRS1 activity will await structural analysis of PRS and its interactions with effector molecules, a model for the relationship between these allosteric mechanisms can be suggested. In this model, nucleotide and Pi alterations of PRS1 activity reflect a shared mechanism in which inhibited conformations favored by nucleotide binding are in equilibrium with more active conformations favored by $\mathrm{Pi}$ binding. Under these circumstances, the various mutant PRS1s would seem likely to be altered in residues that are involved in stabilizing the inhibited conformations, rather than directly in $\mathrm{Pi}$ or nucleotide binding.

\section{Acknowledgments}

We thank Dr. O. Sperling (Tel Aviv University, Israel), Dr. B. Mousson (Hopital Debrousse, Lyon, France), Dr. J. G. Puig and Dr. F. A. Mateos (Hospital LaPaz, Madrid, Spain), Dr. F. Hanefeld and Dr. H. J. Christen (Georg-August-Universitat Gottingen, Germany) for providing fibroblast cultures from their patients, and Dr. Giri A. Reddy, Director of the University of Chicago Amino Acid and Protein Core Laboratory, for performing protein sequence analyses.

This work was supported by National Institutes of Health grants DK-28554, DK-13488, and GM-47712 and by a grant from the Arthritis Foundation, Greater Chicago Chapter.

\section{References}

1. Becker, M. A., K. O. Raivio, and J. E. Seegmiller. 1979. Synthesis of phosphoribosylpyrophosphate in mammalian cells. Adv. Enzymol. Relat. Areas Mol. Biol. 49:281-306.

2. Kornberg, A., I. Lieberman, and E. S. Simms. 1955. Enzymatic synthesis and properties of 5-phosphoribosylpyrophosphate. J. Biol. Chem. 215:389-402.

3. Fox, I. H., and W. N. Kelley. 1971. Human phosphoribosylpyrophosphate synthetase: distribution, purification and properties. J. Biol. Chem. 246:57395748.

4. Fox, I. H., and W. N. Kelley. 1972. Human phosphoribosylpyrophosphate synthetase: kinetic mechanism and end product inhibition. J. Biol. Chem. 247:2126-2131.

5. Willis, R. C., L. D. Nord, J. M. Fujitaki, and R. K. Robins. 1989. Potent and specific inhibitors of mammalian phosphoribosylpyrophosphate (PRPP) synthetase. Adv. Enzyme Regul. 28:167-182.

6. Balzarini, J., and E. DeClercq. 1991. 5-phosphoribosyl 1-pyrophosphate synthetase converts the acyclic nucleoside phosphonates 9-(3-hydroxy-2-phosphonylmethoxypropyl) adenine and 9-(2-phosphonylmethoxyethyl) adenine directly to their antivirally active diphosphate derivatives. J. Biol. Chem. 266:86868689.

7. Yen, R. C. K., W. B. Adams, C. Lazar, and M. A. Becker. 1978. Evidence for X-linkage of human phosphoribosylpyrophosphate synthetase. Proc. Natl. Acad. Sci. USA. 75:482-485.

8. Sperling, O., P. Boer, S. Persky-Brosh, E. Kanarek, and A. deVries. 1972. Altered kinetic property of erythrocyte phosphoribosylpyrophosphate synthetase in excessive purine production. Rev. Eur. Etud. Clin. Biol. 17:703-706.

9. Zoref, E., A. DeVries, and O. Sperling. 1975. Mutant feedback-resistant phosphoribosylpyrophosphate synthetase associated with purine overproduction and gout. J. Clin. Invest. 56:1093-1099.

10. Becker, M. A., M. J. Losman, and M. Kim. 1987. Mechanisms of accelerated purine nucleotide synthesis in human fibroblasts with superactive phosphoribosylpyrophosphate synthetases. J. Biol. Chem. 262:5596-5602.

11. Sperling, O., S. Persky-Brosh, P. Boer, and A. deVries. 1973. Human erythrocyte phosphoribosylpyrophosphate synthetase mutationally altered in regulatory properties. Biochem. Med. 7:389-395.

12. Becker, M. A., J. G. Puig, F. A. Mateos, M. L. Jiminez, M. Kim, and H. A. Simmonds. 1988. Inherited superactivity of phosphoribosylpyrophosphate synthetase: association of uric acid overproduction and sensorineural deafness Am. J. Med. 85:383-390.

13. Becker, M. A., M. J. Losman, J. Wilson, and H. A. Simmonds. 1986 Superactivity of phosphoribosylpyrophosphate synthetase due to altered regulation by nucleotide inhibitors and inorganic phosphate. Biochim. Biophys. Acta. 882:168-176.

14. Lejeune, E., M. Bouvier, B. Mousson, G. Llorca, and P. Baltassat. 1979. Anomalies de la phosphoribosylpyrophosphate synthetase dans deux cas de goutte a debout precoce. Rev. Rhum. Mal. Osteoartic. 46:457-465.

15. Becker, M. A., P. J. Kostel, and L. J. Meyer. 1975. Human phosphoribosylpyrophosphate synthetase. Comparison of purified normal and mutant enzymes. J. Biol. Chem. 250:6822-6830.

16. Becker, M. A., M. J. Losman, P. Itkin, and P. A. Simkin. 1982. Gout with superactive phosphoribosylpyrophosphate synthetase due to increased enzyme catalytic rate. J. Lab. Clin. Med. 99:485-511.

17. Becker, M. A., M. J. Losman, A. L. Rosenberg, I. Mehlman, D. J. Levinson, and E. W. Holmes. 1986. Phosphoribosylpyrophosphate synthetase superactivity. A study of five patients with catalytic defects in the enzyme. Arthritis Rheum. 29:880-888.

18. Akaoka, I., S. Fujimori, N. Kamatani, R. Takeuchi, E. Yano, Y. Nishida, A. Hashimoto, and Y. Horiuchi. 1981. A gouty family with increased phosphoribosylpyrophosphate synthetase activity: case reports, family studies, and kinetic studies of the abnormal enzyme. J. Rheumatol. 8:563-574.

19. Nishida, Y., I. Akaoki, and Y. Horiuchi. 1981. Altered isoelectric property of a superactive 5-phosphoribosyl-1-pyrophosphate (PRPP) synthetase in a patient with clinical gout. Biochem. Med. 26:387-394.

20. Becker, M. A., K. O. Raivio, B. Bakay, W. B. Adams, and W. L. Nyhan. 1980. Variant human phosphoribosylpyrophosphate synthetase altered in regulatory and catalytic functions. J. Clin. Invest. 65:109-120.

21. Losman, M. J., D. Rimon, M. Kim, and M. A. Becker. 1985. Selective expression of phosphoribosylpyrophosphate synthetase superactivity in human lymphoblast lines. J. Clin. Invest. 76:1657-1664.

22. Becker, M. A. 1976. Patterns of phosphoribosylpyrophosphate and ribose5-phosphate concentration and generation in fibroblasts from patients with gout and purine overproduction. J. Clin. Invest. 57:308-318.

23. Simmonds, H. A., D. R. Webster, S. Lingham, and J. Wilson. 1985. An inborn error of purine metabolism, deafness and neurodevelopmental abnormality Neuropediatrics. 16:106-108.

24. Rosenberg, A. L.,L. Bergstrom, B. T. Troost, and B. A. Bartholomew. 1970. Hyperuricemia and neurologic defects: a family study. N. Engl. J. Med. 282:992-997.

25. Taira, M., J. Kudoh, S. Minoshima, T. Iizasa, H. Shimada, Y. Shimizu, M. Tatibana, and N. Shimizu. 1989. Localization of human phosphoribosylpyrophosphate synthetase subunit I and II genes (PRPS1 and PRPS2) to different regions of the X chromosome and assignment of two PRPS-related genes to autosomes. Somatic Cell Mol. Genet. 15:29-37.

26. Becker, M. A., S. A. Heidler, G. I. Bell, S. Seino, M. M. LeBeau, C. A. Westbrook, W. Neuman, L. J. Shapiro, T. K. Mohandas, B. J. Roessler, and T. D. Palella. 1990. Cloning of cDNAs for human phosphoribosylpyrophosphate 
synthetase 1 and 2 and X chromosome localization of PRPS1 and PRPS2 genes. Genomics. 8:555-561.

27. Sonoda, T., M. Taira, S. Ishijima, T. Ishizuka, T. Iizasa, and M. Tatibana. 1991. Complete nucleotide sequence of human phosphoribosylpyrophosphate synthetase subunit I (PRSI) cDNA and a comparison with human and rat PRPS gene families. J. Biochem. 109:361-364.

28. Iizasa, T., M. Taira, H. Shimada, S. Ishijima, and M. Tatibana. 1989. Molecular cloning and sequencing of human cDNA for phosphoribosylpyrophosphate synthetase subunit II. FEBS Lett. 244:47-50.

29. Ishizuka, T., T. Iizasa, M. Taira, S. Ishijima, T. Sonoda, H. Shimada N. Nagatake, and M. Tatibana. 1992. Promoter regions of the human X-linked housekeeping genes PRPS1 and PRPS2 encoding phosphoribosylpyrophosphat synthetase subunit I and II isoforms. Biochim. Biophys. Acta. 1130:139-148.

30. Taira, M., T. lizasa, K. Yamada, H. Shimada, and M. Tatibana. 1989. Tissue-differential expression of two distinct genes for phosphoribosylpyrophosphate synthetase and existence of the testes-specific transcript. Biochim. Biophys. Acta. 1007:203-208.

31. Nosal, J. M., R. L. Switzer, and M. A. Becker. 1993. Overexpression, purification, and characterization of recombinant human 5-phosphoribosyl-1-pyrophosphate synthetase isozymes I and II. J. Biol. Chem. 268:10168-10175.

32. Roessler, B. J., J. M. Nosal, P. R. Smith, S. A. Heidler, T. D. Palella, R. L Switzer, and M. A. Becker. 1993. Human X-linked phosphoribosylpyrophosphate synthetase superactivity is associated with distinct point mutations in the PRPS1 gene. J. Biol. Chem. 268:26478-26481.

33. Chomczynski, P., and N. Sacchi. 1987. Single-step method of RNA isolation by acid guanidinium thiocyanate-phenol-chloroform extraction. Anal. Biochem. 162:156-159.

34. Ausubel, F. M., R. Brent, R. E. Kingston, D. D. Moore, J. G. Seidman, J. A. Smith, and K. Struhl, editors. 1987. Current Protocols in Molecular Biology John Wiley \& Sons, New York.

35. Davidson, B. L., S. A. Tarle, M. VanAntwerp, D. A. Gibbs, R. W. E. Watts, W. N. Kelley, and T. D. Palella. 1991. Identification of seventeen independent mutations responsible for human hypoxanthine-guanine phosphoribosyltransferase (HPRT) deficiency. Am. J. Hum. Genet. 48:951-958.
36. Studier, R. W., A. H. Rosenberg, J. J. Dunn, and J. S. Dubendorff. 1990 Use of $\mathrm{T}_{7}$ RNA polymerase to direct the expression of cloned genes. Methods Enzymol. 185:60-89.

37. Tabor, S., and C. C. Richardson. 1985. A bacteriophage $T_{7}$ RNA polymerase/promoter system for controlled exclusive expression of specific genes. Proc. Natl. Acad. Sci. USA. 82:1074-1078.

38. Laemmli, U. K. 1970. Cleavage of structural proteins during assembly of the head of bacteriophage $T_{4}$. Nature (Lond.) 227:680-685.

39. O'Farrell, P. H. 1975. High resolution two-dimensional electrophoresis of proteins. J. Biol. Chem. 250:4007-4021.

40. Fry, D. W., M. A. Becker, and R. L. Switzer. 1995. Inhibition of human 5-phosphoribosyl-1-pyrophosphate synthetase by 4-amino-8-[B-D-ribofuranosylamino]pyrimido-[5,4-d] pyrimidine-5'-monophosphate: evidence for interaction at the adenosine-5'-diphosphate allosteric site. Mol. Pharmacol. 47:810-815.

41. Lowry, O. H., N. J. Rosebrough, A. L. Farr, and R. J. Randall. 1951. Protein measurement with the Folin phenol reagent. J. Biol. Chem. 193:1839 1845.

42. Ishijima, S., K. Kita, I. Ahmad, T. Ishizuka, M. Taira, and M. Tatibana. 1991. Expression of rat phosphoribosylpyrophosphate synthetase subunits I and II in Escherichia coli. Isolation and characterization of the recombinant isoforms. J. Biol. Chem. 266:15693-15697.

43. Fersht, A. 1984. Enzyme Structure and Mechanism. Second Edition. W. H Freeman and Company, New York. 272-278.

44. Zoref, E., A. deVries, and O. Sperling. 1976. Metabolic cooperation between human fibroblasts with normal and with superactive phosphoribosylpyrophosphate synthetase. Nature (Lond.) 260:786-788.

45. Taira, M., S. Ishijima, K. Kita, K. Yamada, T. Iizasa, and M. Tatibana 1987. Nucleotide and deduced amino acid sequences of two distinct cDNAs fo rat phosphoribosylpyrophosphate synthetase. J. Biol. Chem. 262:14867-14870.

46. Ahmad, I., S. Ishijima, K. Kita, and M. Tatibana. 1994. Identification of amino-acid residues linked to different properties of phosphoribosylpyrophosphate synthetase isoforms I and II. Biochim. Biophys. Acta. 1207:126-133. 